\title{
Auto-detection of arch curve from dental meshes
}

\author{
Ting $\mathrm{WU}^{1, \mathrm{a}}$, Libing ZHANG ${ }^{1, \mathrm{~b}}$ \\ ${ }^{1}$ College of Mechanical and Electrical Engineering, Jiaxing University, Jiaxing, 314001, China \\ aemail: wuting628@163.com, bemail: libinzhan@126.com
}

Keywords: dental arch, triangular meshes, feature extraction, skeleton line, B-spline fitting

\begin{abstract}
Building the form of dental arch is of great importance for orthodontic diagnosis and treatment. This paper presents a novel automated method for detecting the arch curve from dental triangular meshes with minimal user intervention. The feature region between gingiva and teeth were firstly extracted based on discrete curvature analysis. Then using the feature region as reference, a feature image were generated where the arch skeleton is detected. The arch curve were finally computed to approximate the skeleton line based on B-spline extended fitting. The experiment results demonstrate that the method is fast and robust even in the presence of malocclusion.
\end{abstract}

\section{Introduction}

In orthodontics, dental arch plays an important role in measuring dental alignment and dental space whether the teeth are at the appropriated positions and space [1]. Therefore, a complete description of the form of dental arch is essential whenever changes due to growth or orthodontic therapies are under investigation. However, due to the large variation in the shape of the archform among humans, it is difficult to formulate a single mathematical expression to describe all archforms.

With the rapid development of computer technology in stomatology, many computer-aided approaches have been presented to detect archform. Sinthanayothin et al. [2] interactively selected feature points on the model to fit the dental arch. Kondo et al. [3] presented a two-step curve fitting method to obtain the dental arch. They first detected the tooth cusps and ridges using gradient orientation analysis on the plan-view range image; these cusps and ridges were then used to fit an initial polynomial curve; along this curve the perpendicular spokes were set up to detect the orthodontic features necessary for determining the final higher order polynomial arch curve. Kumar et al. [4] used the experimentally derived equation in [5] to compute the initial approximation to the archform based on the searched tooth features; the archform was then presented as a B-spline curve so that users can modify it using the control points along the curve.

In this paper, we propose a novel approach that employs curvature analysis, morphology operation and B-spline fitting technology to automatically estimate dental arch. The method can be applied to handle various cases of malocclusion.

\section{Feature Region Extraction}

As the interface between gingiva and teeth can reflect the teeth arrangement shape along the dental arch, we can extract this region based on curvature information. We use a local cubic surface fitting method [6] to estimate the discrete curvature of the dental model. The method need to establish a local coordinate system at each mesh vertex $\boldsymbol{p}$. Under this local coordinate system, the adjacent vertices coordinates and corresponding normal vectors are used to create a third degree equation for surface fitting:

$$
f(x, y)=\frac{A}{2} x^{2}+B x y+\frac{C}{2} y^{2}+D x^{3}+E x^{2} y+F x y^{2}+G y^{3}
$$


In the least-squares solution, the surface coefficients $(A, B, C, D, E, F, G)^{T}$ can be easily solved. Then based on the surface basic formulas, the maximum and minimum principle curvature value of the vertex $\boldsymbol{p}$ can be deduced as:

$$
\kappa_{\max }=H+\sqrt{H^{2}-K}, \quad \kappa_{\text {min }}=H-\sqrt{H^{2}-K}
$$

where

$$
K=A C-B^{2}, \quad H=(A+C) / 2
$$

Fig. 1a shows the maximum principal curvature visualization of the dental model where the highest and lowest curvatures are corresponding to the red and blue color, respectively. With the maximum principal curvature information, the concave characteristics of gingival margin can be easily identified and extracted through curvature thresholding (Fig. 1b). Then based on morphological operation (opening and closing) [7] and connectivity filtering (i.e., more feature vertices are connected), we can acquire the potential boundary vertices between gingiva and teeth (Fig. 1c).
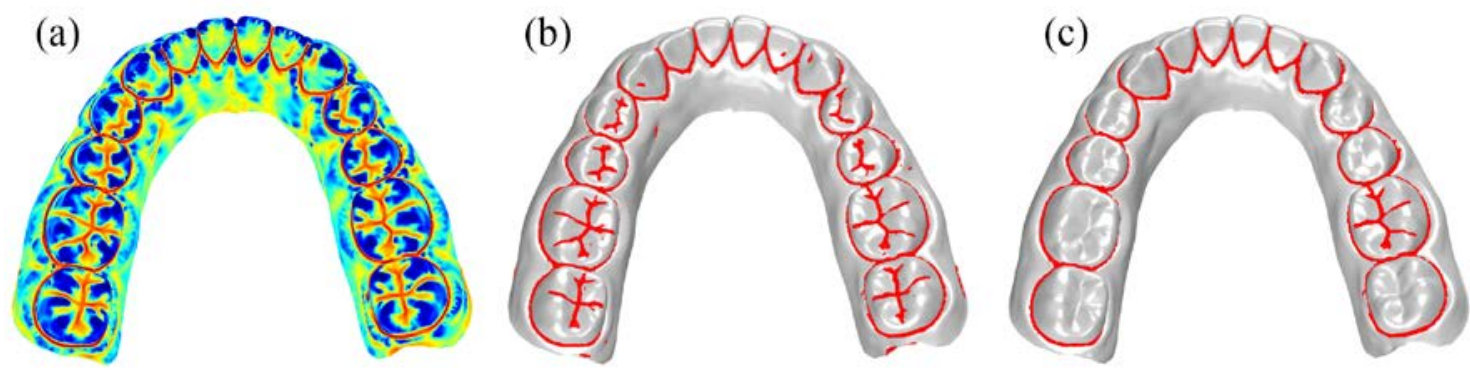

Fig. 1 Feature region extraction: (a) maximum principal curvature visualization; (b) curvature thresholding; (c) final feature region after morphological operation and connectivity filtering.

\section{Arch skeleton Detection}

Once the feature region between gingiva and teeth has been acquired, we can obtain a feature binary Image $I$ (Fig. 2a) by projecting and rounding the vertices of feature region on the occlusal plane,. Through morphological dilation and filling, we can get the Image $I_{\text {fill }}$ where possible gaps and holes inside the teeth are effectively filled (Fig. 2b). Finally by a continuous thinning process to the image $I_{\text {fill }}$, we can get a skeleton line which provide a crude dental arch form (Fig. 2c).

(a)

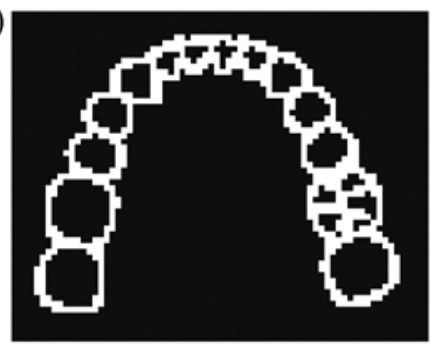

(b)



(c)

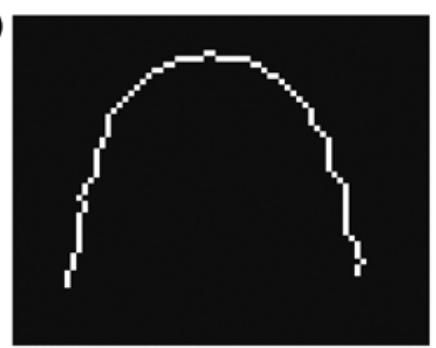

Fig. 2 Arch skeleton detection: (a) binary image generated from feature region; (b) morphology dilation and filling; (c) arch skeleton after morphological thinning

\section{B-spline extended fitting}

With the arch skeleton, we fit them to a cubic B-spline curve. Consider a set of arch skeleton points $\left\{\boldsymbol{Q}_{i}, i=1,2, \ldots, m\right\}$ and a B-spline curve $C(t)$ expressed as:

$$
\boldsymbol{C}(t)=\sum_{j=1}^{n} \boldsymbol{V}_{j} B_{j, p}(t)
$$


where $\left\{\boldsymbol{V}_{j}, j=1,2, \ldots n\right\}$ are the control points, $t$ is the parametric value, and $B_{i, p}(t)$ are the normalized B-spline basis functions defined on the knot vector $\boldsymbol{U}$. The curve $C(t)$ is computed to approximate the points $\left\{\boldsymbol{Q}_{i}\right\}$ by minimizing energy function:

$$
\begin{aligned}
& E=\sum_{i=1}^{m}\left\|\boldsymbol{C}(t)-\boldsymbol{Q}_{i}\right\|^{2}+\lambda \sum_{i=1}^{m}\left\|\boldsymbol{C}^{\prime \prime}(t)\right\|^{2} \Delta t \\
& =\sum_{i=1}^{m}\left\|\sum_{j=1}^{n} \boldsymbol{V}_{j} B_{j, p}\left(t_{i}\right)-\boldsymbol{Q}_{i}\right\|^{2}+\lambda \Delta t \sum_{i=1}^{m-1}\left\|\sum_{j=1}^{n} \boldsymbol{V}_{j} B_{j, p}^{\prime \prime}\left(t_{i}\right)\right\|^{2}
\end{aligned}
$$

where $\lambda$ are weight coefficient, and $\Delta t$ represents the points interval in the parametric space.

In order to make the arch curve cover the full range of the teeth profile, we propose an extended curve fitting method. Suppose the extension ratios on both ends of the curve are $\alpha_{0}$ and $\alpha_{\mathrm{m}}, L$ is the total chordal length:

$$
L=\sum_{i=2}^{m}\left\|\boldsymbol{Q}_{i}-\boldsymbol{Q}_{i-1}\right\|
$$

Therefore the total length of the extended curve can be expressed as:

$$
L_{\text {extend }}=L\left(1+\alpha_{0}+\alpha_{m}\right)
$$

Thus, the parameter $t_{j}$ is computed based on the following equation:

$$
\left\{\begin{array}{l}
t_{1}=\frac{\alpha_{0}}{1+\alpha_{0}+\alpha_{m}}, \\
t_{i}=t_{i-1}+\frac{\left\|\boldsymbol{Q}_{i}-\boldsymbol{Q}_{i-1}\right\|}{L_{\text {extend }}}, \quad i=2,3 \ldots, m
\end{array}\right.
$$

At the convenience of adjustment, we use fewer control points (usually set $n=9$ ), thus the knot vector $\boldsymbol{U}$ can be defined as [8]:

$$
\left\{\begin{array}{l}
u_{1}=u_{2}=\ldots=u_{p+1}=0 \\
u_{p+j}=(1-\alpha) t_{i-1}+\alpha t_{i}, \quad j=2,3, \ldots n-p+1 \\
u_{n+2}=u_{n+3}=\ldots=u_{n+p+1}=1
\end{array}\right.
$$

where $i=\operatorname{int}(j c), c=(m+1) /(n-k+1), \alpha=j c-i$.Then by minimizing the equation (5), we can get the following matrix equation:

$$
\left(\boldsymbol{A}^{T} \boldsymbol{A}+\lambda \Delta t \ddot{\boldsymbol{A}}^{T} \ddot{\boldsymbol{A}}\right) \boldsymbol{V}=\boldsymbol{A}^{T} \boldsymbol{Q}
$$

where

$$
\begin{aligned}
& \boldsymbol{A}=\left[\begin{array}{cccc}
B_{1, p}\left(t_{1}\right) & B_{2, p}\left(t_{1}\right) & \cdots & B_{n, p}\left(t_{1}\right) \\
B_{1, p}\left(t_{2}\right) & B_{2, p}\left(t_{2}\right) & \cdots & B_{n, p}\left(t_{2}\right) \\
\vdots & \vdots & \ddots & \vdots \\
B_{1, p}\left(t_{m}\right) & B_{2, p}\left(t_{m}\right) & \cdots & B_{n, p}\left(t_{m}\right)
\end{array}\right], \quad \ddot{\boldsymbol{A}}=\left[\begin{array}{cccc}
B_{1, p}^{\prime \prime}\left(t_{1}\right) & B_{2, p}^{\prime \prime}\left(t_{1}\right) & \cdots & B_{n, p}^{\prime \prime}\left(t_{1}\right) \\
B_{1, p}^{\prime \prime}\left(t_{2}\right) & B_{2, p}^{\prime \prime}\left(t_{2}\right) & \cdots & B_{n, p}^{\prime \prime}\left(t_{2}\right) \\
\vdots & \vdots & \ddots & \vdots \\
B_{1, p}^{\prime \prime}\left(t_{m}\right) & B_{2, p}^{\prime \prime}\left(t_{m}\right) & \cdots & B_{n, p}^{\prime \prime}\left(t_{m}\right)
\end{array}\right] \\
& \boldsymbol{V}=\left[\begin{array}{llll}
\boldsymbol{V}_{1} & \boldsymbol{V}_{2} & \ldots & \boldsymbol{V}_{n}
\end{array}\right]^{T} \quad \boldsymbol{Q}=\left[\begin{array}{llll}
\boldsymbol{Q}_{1} & \boldsymbol{Q}_{2} & \ldots & \boldsymbol{Q}_{m}
\end{array}\right]^{T}
\end{aligned}
$$

Moreover, we add two constrained conditions to yield natural extending status:

$$
\left\{\begin{array} { l } 
{ \boldsymbol { C } ^ { \prime \prime } ( 0 ) = \mathbf { 0 } } \\
{ \boldsymbol { C } ^ { \prime \prime } ( 1 ) = \mathbf { 0 } }
\end{array} \Rightarrow \left\{\begin{array}{l}
\sum_{j=1}^{n} \boldsymbol{V}_{j} B^{\prime \prime}{ }_{j, p}(0)=\mathbf{0} \\
\sum_{j=1}^{n} \boldsymbol{V}_{j} B_{j, p}(1)=\mathbf{0}
\end{array}\right.\right.
$$

Equation (12) can further be expressed as a matrix form as follows: 


$$
N_{a} \boldsymbol{V}=\mathbf{0}
$$

where

$$
\boldsymbol{N}_{a}=\left[\begin{array}{cccc}
B_{1, p}^{\prime \prime}(0) & B_{2, p}^{\prime \prime}(0) & \cdots & B_{n, p}^{\prime \prime}(0) \\
B_{1, p}^{\prime \prime}(1) & B_{2, p}^{\prime \prime}(1) & \cdots & B_{n, p}^{\prime \prime}(1)
\end{array}\right]_{2 \times n}
$$

By combining Eq. (10) and Eq. (13), we can get a set of simultaneous equations as follows:

$$
\left[\begin{array}{cc}
\left(\boldsymbol{A}^{T} \boldsymbol{A}+\lambda \Delta t \ddot{\boldsymbol{A}}^{T} \ddot{\boldsymbol{A}}\right) & \boldsymbol{N}_{a}^{T} \\
\boldsymbol{N}_{a} & \mathbf{0}
\end{array}\right]\left[\begin{array}{c}
\boldsymbol{V} \\
\boldsymbol{\Lambda}
\end{array}\right]=\left[\begin{array}{c}
\boldsymbol{A}^{T} \boldsymbol{Q} \\
\mathbf{0}
\end{array}\right]
$$

where $\Lambda$ is the vector storing Lagrange multiples. Finally through the least-squares method, the control points $\left\{\boldsymbol{V}_{j}\right\}$ can be easily solved. Figure 3 displays the fitted arch curve with standard fitting and extended fitting as well as the difference between with and without end constraint.

(a)

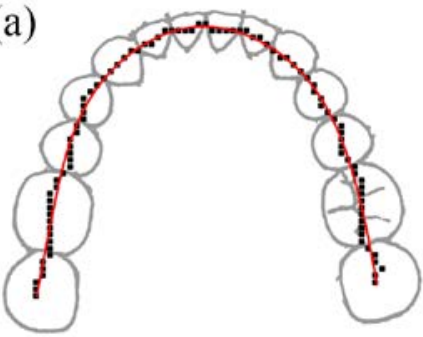

(b)

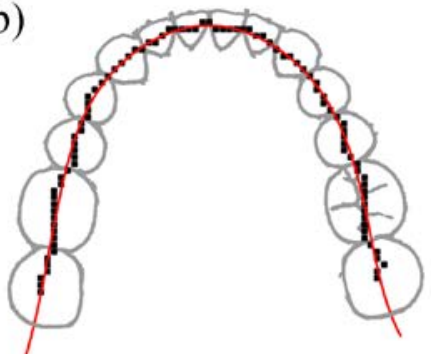

(c)



Fig. 3 Dental arch curve fitting: (a) standard fitting; (b) extended fitting without constraint; (c) extended fitting with constraint

\section{Experiments and Discussions}

The proposed method was tested on 10 different dental models of varying complexity. These dental models were acquired by a commercially available optical scanner, each with approximately $0.15 \mathrm{~mm}$ vertex spacing that can meet the clinical precision requirement. Our system performed with reasonable accuracy on most of the models, requiring user interaction only to select the reference points to define occlusal plane.

Fig. 4 shows the arch fitting process of two different patient models. Model 1 and Model 2 are both complex models with noise and malocclusion. It can be seen that our method can produce good arch curves that accurately reflect the tooth arrangement tendency even in the presence of distorted tooth shapes. These results could assists the clinicians for the treatment planning and the patients can also get an overall idea of what will affect to their teeth after the orthodontics treatment.
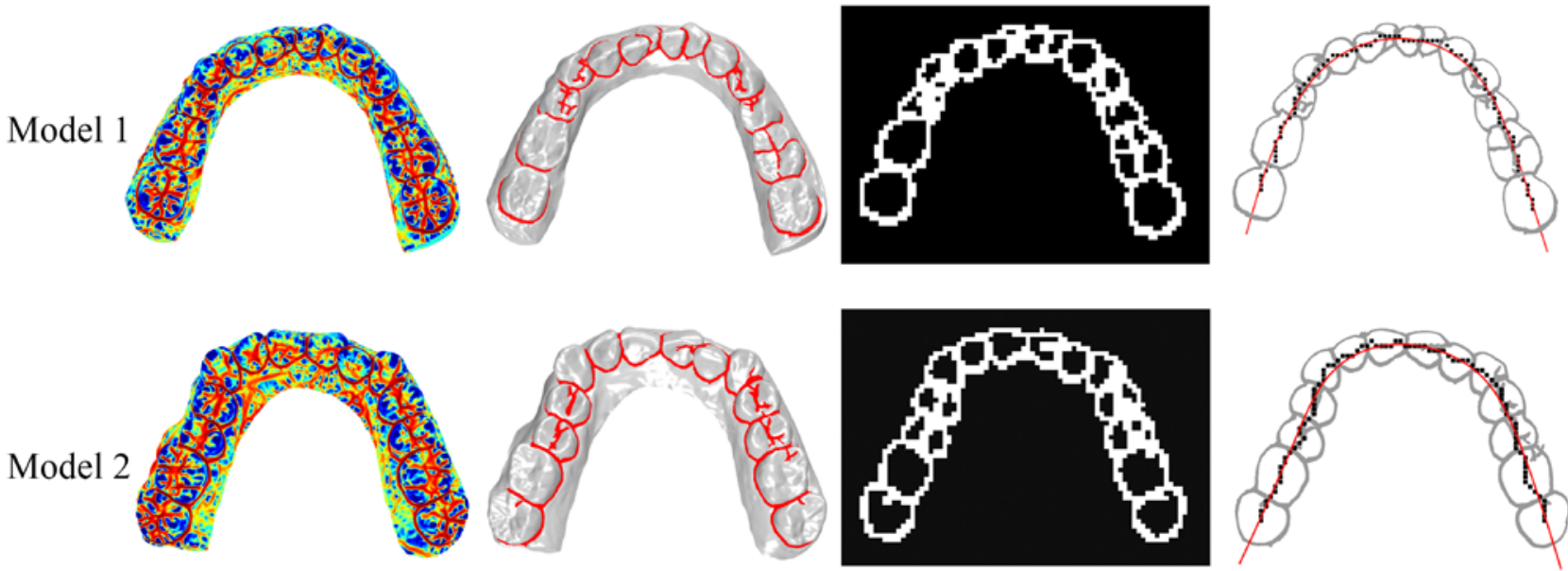

Fig. 4 Examples of dental arch fitting on the experimental models shown in rows. 


\section{Conclusions}

This study presented a highly automated arch detecting method from dental triangular meshes. The algorithm is based on discrete curvature analysis, morphology operation and B-spline fitting technology. Through our experiments, we found that our approach is fast and requires minimal user interaction, and is robust even in the presence of heavy noise and malocclusion. Hence it enables orthodontics to plan dental treatments much faster.

The fitted arch curve can be used for setting planning treatments and recording archform variations of patients. However, our whole system is still in experimental setup phase at present, so the evaluation of its reliability was preliminary. In the future, further refinement and quantitative clinic experiments will be carried out in order to improve and practice this system.

\section{Acknowledgements}

The study was financially supported by the Zhejiang Provincial Natural Science Foundation of China (Grant No.: LQ14E050006) and the National Natural Science Foundation of China (Grant No.: 51405197).

\section{References}

[1] Wisarut Bholsithi, Chanjira Sinthanayothin. Digital Dental Model Analysis. Proceedings of the 13th International Conference on Biomedical Engineering [C], 2008, 23: 472-475.

[2] Chanjira Sinthanayothin, Wichit Tharanont. Orthodontics Treatment Simulation by Teeth Segmentation and Setup. Proceedings of the 5th International Congress on Electrical Engineering/Electronics, Computer, Telecommunications and Information Technology, 2008, 1: 81-84.

[3] Toshiaki Kondo, Sim Heng Ong, Kelvin W. C. Foong. Tooth Segmentation of Dental Study Models Using Range Images [J]. IEEE Transactions on Medical Imaging, 2004, 23(3): 350-362.

[4] Yokesh Kumar, Ravi Janardan, Brent Larson. Automatic Feature Identification in Dental Meshes [J]. Computer-Aided Design and Applications, 2013, 9 (6): 747-769.

[5] Stanley. Braun, William P. Hnat, Dana E. Fender, Harry L. Legan. The form of the human dental arch [J]. Angle Orthodontist, 1998, 68(1): 29-36.

[6] Jack Goldfeather, Victoria Interrante. A novel cubic-order algorithm for approximating principal direction vectors [J]. ACM Transactions on Graphics, 2004, 23(1): 45-63.

[7] Christian Rössl, Leif Kobbelt, Hans-Peter Seidel. Extraction of feature lines on triangulated surfaces using morphological operators [C]. Proceedings of the AAAI Symposium on Smart Graphics, 2000: 71-75.

[8] Les Piegl, WayneTiller: The NURBS book [M]. Springer Berlin Heidelberg, 1997. 